\title{
Extracranial Carotid Artery Disease
}

Magnetic resonance angiography (MRA) is a noninvasive means by which to study the integrity of the vascular system (Potchen et al., 1992; Anderson et al., 1993).

This unit presents two MRA techniques for imaging the carotid arteries, time-of-flight MRA (see Basic Protocol 1) and contrast-enhanced MRA (see Alternate Protocol). As a complement to MRA, following either of the preceding two procedures, high-resolution spin-echo scanning can be used to study the vessel wall in more detail (see Basic Protocol 2 ). The parameters given here are derived from experience at $1.5 \mathrm{~T}$ and may need to be altered slightly depending on the field strength and the equipment manufacturer. In particular, optimal $T_{\mathrm{E}}$ may vary with different field strength.

\section{IMAGING THE CAROTID ARTERIES BY TIME-OF-FLIGHT MRA}

Magnetic resonance angiography (MRA) scans can be run at a range of field strengths with varying degrees of success. The high signal-to-noise ratio (SNR) obtained at a high-field strength makes it possible to run the MRA scans either faster or with higher resolution than at lower fields. (Low-field strength is generally $\leq 0.2 \mathrm{~T}$, mid-field strength is between $0.5 \mathrm{~T}$ and $1 \mathrm{~T}$, and high-field strength is $\geq 1.5 \mathrm{~T}$.) At low-field strengths, contrast-enhanced MRA (see Alternate Protocol) may give better results than conventional time-of-flight (TOF) MRA. The sequences described herein are based on the authors' experience with a Siemens $1.5 \mathrm{~T}$ Vision scanner, but are expected to be equally applicable to machines from other manufacturers.

The following three sequences comprise the preferred protocol. In cases where a patient requires a faster procedure (e.g., for trauma patients or those intolerant to a long scanning session), either the two-dimensional (2-D) sequence (sequence 2) or the three-dimensional (3-D) sequence (sequence 3) may be omitted. Because the 3-D sequence offers higher through-plane resolution $(1 \mathrm{~mm})$ than the 2-D sequence, some radiologists and researchers have suggested that 3-D techniques are more accurate than 2-D techniques in detecting carotid stenosis. However, in older patients, for whom flow can be very slow, the 2-D sequence may give better results. For patients with tortuous vessels, the contrastenhanced method (see Alternate Protocol) may be most appropriate. If desired, this procedure may be followed by high-resolution spin-echo scanning (see Basic Protocol 2) for a more detailed view of vessel walls. The entire Basic Protocol 1 takes $\sim 40 \mathrm{~min}$.

Table A1.3.1 lists the hardware necessary to perform the procedure, along with appropriate parameters. The available gradient strength will depend on the scanner, and the echo

Table A1.3.1 Equipment Parameters for Time-of-Flight MRA

\begin{tabular}{ll}
\hline Coil type & $\begin{array}{l}\text { Circularly polarized neck coil or } \\
\text { neuro-vascular coil (or phased-array neck } \\
\text { coil, if available) } \\
\end{array}$ \\
$\begin{array}{l}\text { Gradient coil strength } \\
\text { Cardiac gating }\end{array}$ & No \\
Peripheral gating & For safety only \\
Respiratory gating & No \\
Respirator & If required by patient \\
Oxygen & If required by patient \\
Motion cushions & Useful \\
Use of contrast agents & Only use in the Alternate Protocol \\
\hline
\end{tabular}

Contributed by E. Mark Haacke, Daniel Kido, and Karen Tong

Current Protocols in Magnetic Resonance Imaging (2002) A1.3.1-A1.3.13

Copyright (C) 2002 by John Wiley \& Sons, Inc.
BASIC

PROTOCOL 1

Intracranial Arterial Disease

A1.3.1

Supplement 7 
times given below may be varied accordingly (the lower the gradient strength, the longer the echo time for a particular scan).

NOTE: Be sure that technologists and nurses have immediate access to any emergency equipment that may be relevant to a given study, or that may be needed for a particular patient, such as crash carts or oxygen.

\section{Set up patient and equipment}

1. Interview (screen) the patient to ensure that he or she has no contraindications such as cardiac pacemakers or other implants containing ferromagnetic materials that may be problematic for patient safety or good image acquisition. Also be sure to find out if the patient has any health conditions that may require the presence of special emergency equipment during the scanning procedure, or necessitate any other precautions.

Generally, standard screening forms (APPENDIX 1) are used for all patients scanned in a magnetic resonance system.

The presence of any ferromagnetic metals may be a health hazard to the patient when he or she is inside the magnet, and will also affect the imaging. If in doubt as to the exact composition of the items, it is best to exclude patients with any metal implants; see Shellock (2001) for a discussion of what implants may be safely scanned using magnetic resonance.

Patients may be accompanied into the magnet room by a friend or family member, who can sit in the room during the scan and comfort the patient as needed. This companion must be screened as well to ensure the absence of loose metal objects on the body or clothing, as well as other items as described above.

2. If the procedure is a research protocol, have the patient sign any necessary consent forms.

3. Have the patient remove all jewelry and change into a gown to eliminate any metal that might be found in clothing.

4. Have the patient wash off any mascara and other makeup to avoid local tissue heating and image artifacts.

5. Inform the patient about what will occur during the procedure, what he or she will experience while in the magnet, and how to behave, including the following:

a. If earplugs, earphones, or headphones are used to protect the ears from the loud sounds produced by the gradients, the patient will be asked to wear one of these devices, but will be able to communicate with you at any time during the imaging.

b. The patient may be given a safety squeeze-bulb or similar equipment to request assistance at any time (demonstrate how this works).

c. For good results, the patient should not talk, and should avoid or minimize swallowing or other movement during each scan-i.e., as long as the banging sounds continue. Between scans, talking and swallowing are allowed in most cases, but should be avoided when comparative positional studies are being performed; the patient will be informed when this is the case.

d. Nevertheless, the patient may call out at any time if he or she feels it necessary.

6. Have the patient mount onto the table. Either before or right after the patient lies down, set up any triggering devices or other monitoring equipment that is to be used. 
7. Center the patient in a neck coil, or a neuro-vascular coil, at the region where the key information is desired. Make sure that the head and neck are constrained to prevent motion, especially if high-resolution scans are to be run.

Generally, the patient's head and neck are fixed so that they are horizontal (not tilted) and lie along the axis of the patient table; other positions may be appropriate depending on the imaging needs.

Most scanners have a special neck coil or a neuro-vascular coil for MRA; otherwise, a head coil and/or cervical spine coil should be used and the patient placed so that the bifurcation of the main carotid artery into the internal and external carotid arteries can be imaged.

8. If needed, place a pillow or other support under the knees to make the patient more comfortable.

9. Use the centering light to position the patient at the angle of the mandible and put the patient into the center of the magnet.

Once this step has been performed, so long as the patient does not move on the table, the table itself can be moved and then returned to the same position as before without jeopardizing the positioning of one scan relative to another.

10. If the patient is unable to hold still, provide an appropriate sedative.

\section{Sequence 1: Rapid three-plane positioning pilot}

11. To validate the patient's position, run the system's pilot (or scout) scan to ensure correct location of the neck in three dimensions using the imaging sequence given in Table A1.3.2 or similar parameters. Refer to manufacturer specifications for standard default settings and swap as necessary.

This sequence usually consists of three orthogonal planes to allow localization. The images are often also used later to determine where to place the saturation pulses and to set up total coverage of the volume of interest.

\section{Sequence 2: 2-D time-of-flight MRA}

12. Bring the sequence for a rapid 2-D transverse scan up onto the console. Set the imaging parameters as shown in Table A1.3.3.

13. Use the pilot (scout) images run earlier (sequence 1) to locate the vessel positions in three dimensions and ensure coverage of the region of interest. Specifically, make sure the carotid bifurcation is in the center of the field of view. Use the pilot images to set up either a saturation pulse caudally to suppress signal from the incoming arterial flow or cephalically (cranially) to suppress signal from the incoming venous flow (see Fig. A1.3.1).

See Potchen et al. (1992), Anderson et al. (1993), and Haacke et al. (1999) for details of setting up a saturation pulse.

The system usually applies this as a traveling saturation pulse to continue to saturate venous flow near the plane being imaged but not so close as to interfere with application of the usual slice selection process.

14. Let the patient know you are ready, and begin the scan.

\section{Data processing and viewing for sequence 2}

15. If a better view of the images is necessary, modify the window and level settings and obtain a close-up view of a region by zooming in on the data (images). For example, if a stenosis appears in the image that needs to be measured, zoom in on the image by at least a factor of 2 and then use the distance function in the manufacturer's software to determine the width of the stenosis. This can then be compared to a more normal portion of the vessel, if possible, either distal to the stenosis (NASCET criteria) or proximal to the stenosis.

Intracranial Arterial Disease

A1.3.3

Supplement 7 
Table A1.3.2 Primary Clinical Imaging Parameters for Sequence 1 (Pilot Scan)

\begin{tabular}{ll}
\hline Patient position & Supine \\
Scan type & 3-D gradient echo \\
Imaging plane (orientation) & Sagittal/coronal/transverse (many \\
& scanners are now capable of triple \\
plane orthogonal scouts)
\end{tabular}

Table A1.3.3 Primary Clinical Imaging Parameters for Sequence 2 (2-D TOF)

Patient position

Scan type

Imaging plane (orientation)

Central slice or volume center

Echo time $\left(T_{\mathrm{E}}\right)$

Repeat time $\left(T_{\mathrm{R}}\right)$

Flip angle (FA)

Fields of view $\left(\mathrm{FOV}_{\mathrm{x}}, \mathrm{FOV}_{\mathrm{y}}\right)$

Resolution $(\Delta x, \Delta y)$

Number of data points collected $\left(N_{\mathrm{x}}, N_{\mathrm{y}}\right)$

Display matrix $\left(D_{\mathrm{x}}, D_{\mathrm{y}}\right)$

Slice thickness $(\Delta z)$

Number of slices

Slice gap

Number of acquisitions $\left(N_{\text {acq }}\right)$

Read direction

Slice locations

Saturation pulses

Slice series

Scan time

\section{Supine}

2-D gradient echo

Transverse

Centered on the carotid bifurcation

As short as possible (usually $<7 \mathrm{msec}$ )

$25 \mathrm{msec}$

$35^{\circ}$

$256 \mathrm{~mm}, 160-200 \mathrm{~mm}$

$1 \mathrm{~mm}, 1-1.25 \mathrm{~mm}$ or $0.5 \mathrm{~mm}, 1-1.25$

$\mathrm{mm}$

256,160 or 512,160

512,512

2-3 mm

$50-70$, or as many as needed to cover the region of interest

$-0.33 \mathrm{~mm}$ (overlapping)

1

Anterior-posterior

Carotid bifurcation or stenosis

Yes; cephalically (cranially) thick slab to saturate veins or caudally thick slab to suppress arteries

Ascending or descending, but not interleaved $^{a}$

$\sim 5 \mathrm{~min}$
Extracranial Carotid Artery Disease

A1.3.4

$a_{\text {This will help eliminate reconstruction artifacts. }}$ 


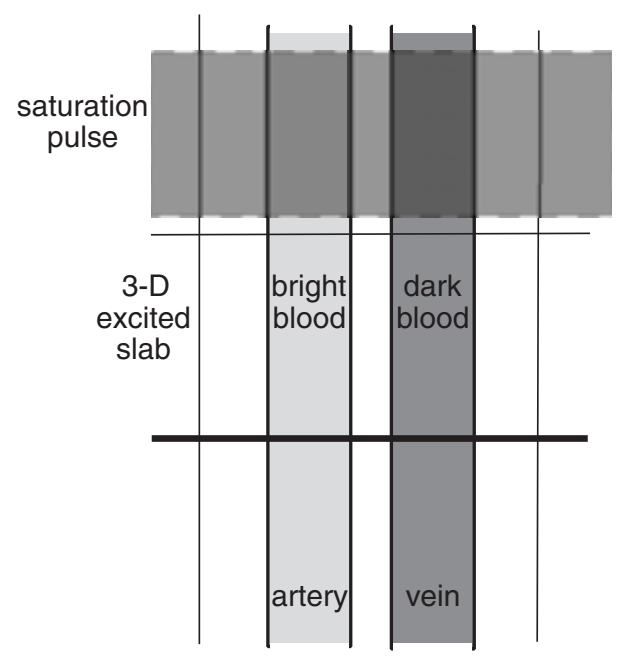

Figure A1.3.1 Schematic of the carotid artery and jugular vein. The images resulting from the 3-D TOF scan (sequence 3; Fig. A1.3.2) show dramatically reduced signal from the veins.

A

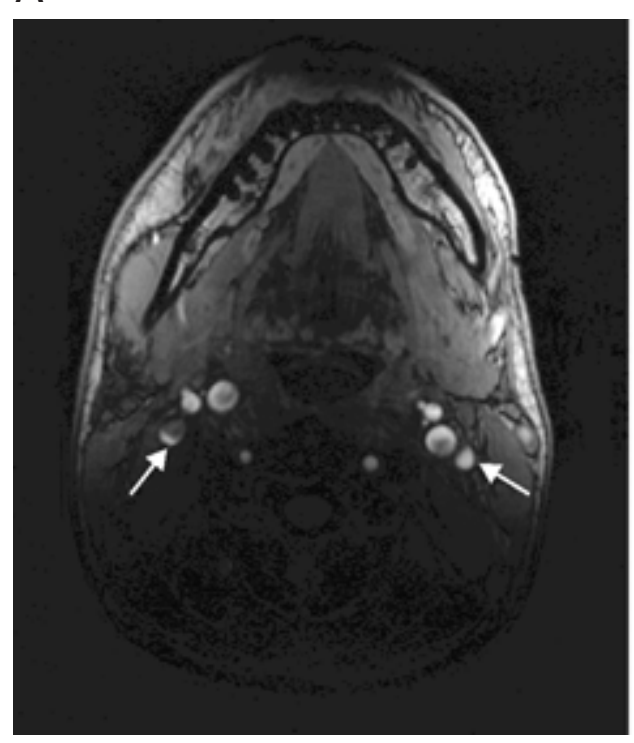

C

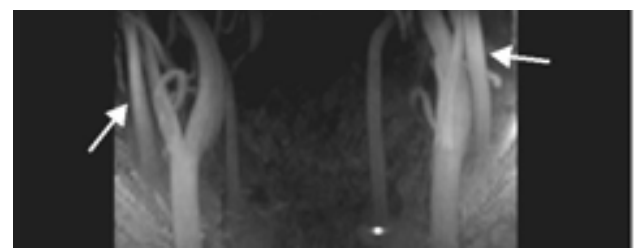

B

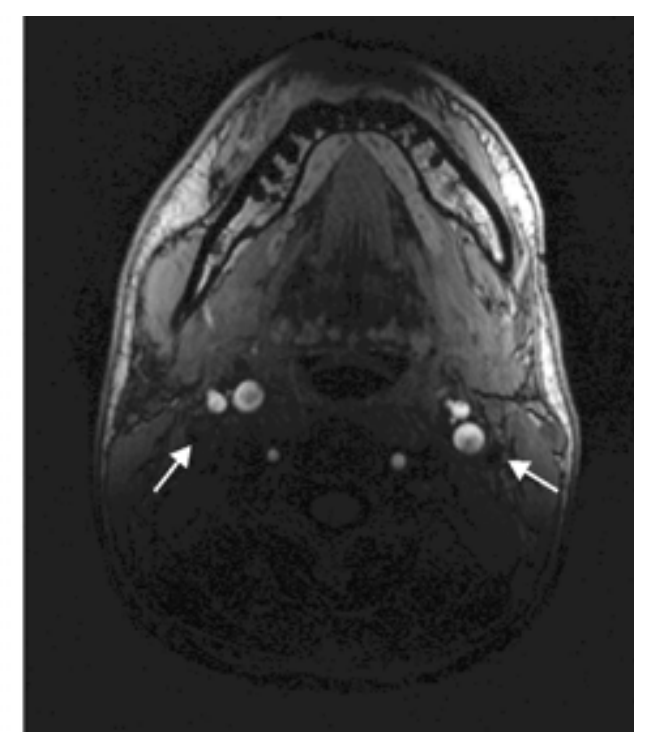

D

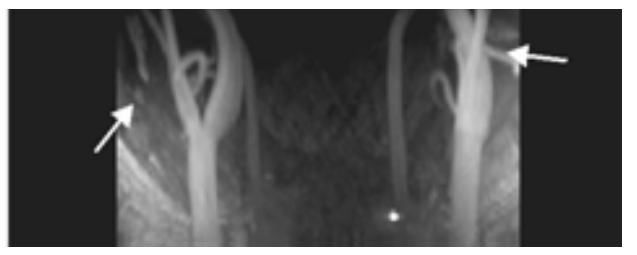

Figure A1.3.2 Transverse and coronal planes of a human neck. (A) A transverse image across the neck without a saturation pulse shows a cross-section of both the carotid artery and the jugular vein (arrows). (B) After a saturation pulse, the signal from the jugular vein is dramatically suppressed. (C, D) Coronally reformatted images obtained from the same 3-D transverse data sets used to obtain the images shown in $(\mathbf{A})$ and $(\mathbf{B})$, respectively. Note that the internal jugular veins (arrows) have vanished in (B) and (D). 
Table A1.3.4 Primary Clinical Imaging Parameters for Sequence 3 (3-D TOF) ${ }^{a}$

\begin{tabular}{|c|c|}
\hline Patient position & Supine \\
\hline Scan type & $3-\mathrm{D}$ gradient echo \\
\hline Imaging plane (orientation) & Transverse \\
\hline Central slice or volume center & Centered on the carotid bifurcation \\
\hline Echo time $\left(T_{\mathrm{E}}\right)$ & $\begin{array}{l}\text { As short as possible; but using } 7 \mathrm{msec} \\
\text { or } 2.5 \mathrm{msec} \text { to } 3.0 \mathrm{msec} \text { ensures that } \\
\text { water and fat are out of phase }\end{array}$ \\
\hline Repeat time $\left(T_{\mathrm{R}}\right)$ & $20-40 \mathrm{msec}$ \\
\hline Flip angle (FA) & $15^{\circ}-25^{\circ}$ \\
\hline Fields of view $\left(\mathrm{FOV}_{\mathrm{x}}, \mathrm{FOV}_{\mathrm{y}}\right)$ & $256 \mathrm{~mm}, 160 \mathrm{~mm}$ \\
\hline Resolution $(\Delta x, \Delta y)$ & $1 \mathrm{~mm}, 0.83 \mathrm{~mm}$ \\
\hline Number of data points collected $\left(N_{\mathrm{x}}, N_{\mathrm{y}}\right)$ & 512,192 \\
\hline Display matrix $\left(D_{\mathrm{x}}, D_{\mathrm{y}}\right)$ & 512,512 \\
\hline Slice thickness $(\Delta z)$ & $1 \mathrm{~mm}$ \\
\hline Number of slices & 64 \\
\hline Slab thickness & $64 \mathrm{~mm}$ \\
\hline Slice gap & $-0.38 \mathrm{~mm}$ (overlapping) \\
\hline Number of acquisitions $\left(N_{\text {acq }}\right)$ & 1 \\
\hline Read direction & Anterior-posterior \\
\hline Slice locations & Carotid bifurcation or stenosis \\
\hline Saturation pulses & $\begin{array}{l}\text { Yes; } 50 \text { to } 80 \mathrm{~mm} \text { thick slabs } \\
\text { cephalically (cranially) to saturate } \\
\text { veins or caudally to suppress arteries }\end{array}$ \\
\hline Fat suppression & Yes \\
\hline Scan time & $\sim 4-8 \mathrm{~min}$ \\
\hline
\end{tabular}

${ }^{a}$ Need RF spoiled pulse.

This zoom feature is only useful if it is done by interpolation of the images, not by pixel duplication. Ideally, this should be done in the in-plane view, but if this is not available or the data cannot be manipulated using multiplanar reformatting, a through-plane view through the center of the vessel can be substituted.

16. Once the scan is complete, process the data as needed to produce a maximum intensity projection (MIP) image (UNIT B7.3) of the vessels at different angles.

The resulting projections can then be viewed as a cine or movie loop to allow the physician to obtain a more global perspective of the vasculature. If there are questions of accuracy associated with this processing, go back and view the pristine data by reexamining the original images or by slicing the original data in a transverse mode (or the view that gives the best cross-sectional information) via a multiplanar reformatting to better view the cross-section of the vessel of interest.

\section{Sequence 3: 3-D time-of-flight MRA (Fig. A1.3.2)}

17. Review the pilot scans and ensure that the saturation pulse is correctly placed above the slab of interest.

18. Run the 3-D scan using parameters as close as possible to those in Table A1.3.4 to obtain high signal from the arteries.

This sequence is usually performed in a transverse mode to benefit from a maximum inflow effect.

19. Process the data from sequence 3 similar to that for sequence 2 (see steps 15 and 16).

Extracranial Carotid Artery Disease

A1.3.6 


\section{IMAGING THE CAROTID ARTERY BY CONTRAST-ENHANCED} TIME-OF-FLIGHT MRA

The set of imaging sequences presented in Basic Protocol 1 often suffices to reveal the necessary angiographic information. However, the accuracy of non-contrast-enhanced MRA is only slightly better than that of an ultrasound. For slow-flow cases, to obtain better coverage or higher resolution, or where speed is of the essence (such as for elderly patients, trauma patients, or patients who are uncooperative), an alternative procedure using a $T_{1}$-reducing contrast agent is preferred (Prince et al., 1997; Wilman et al., 1998). This can be considered a supplementary approach, but in the near future, it is anticipated that this contrast-enhanced (CE) sequence will eventually replace sequences 2 and 3 described in Basic Protocol 1. If desired, this procedure may be followed by high-resolution spin-echo scanning (see Basic Protocol 2) to obtain a more detailed view of vessel walls. The entire Alternate Protocol takes $\sim 25 \mathrm{~min}$.

\section{Materials}

Gadolinium-based MR contrast agent (e.g., Magnevist, Omniscan, or Prohance)

Normal saline $(0.9 \% \mathrm{NaCl})$, sterile

Power injector

\section{Set up patient and equipment}

1. Set up patient as in Basic Protocol 1, steps 1 to 6.

2. Establish an i.v. line from which the contrast agent can be injected, and attach this line securely to the patient so that movement into or out of the magnet will not pull at the patient's arm.

It is preferable to insert the line prior to imaging and to leave the patient in the magnet, so that there is no intervening motion between the scans run before contrast agent injection and those run after injection.

3. Repeat Basic Protocol 1, steps 7 to 10. Depending on the coverage required, use a neck coil, a neuro-vascular coil, a phased-array head and neck coil, or the body coil.

\section{Run pilot scan}

4. Repeat a rapid three-plane positioning pilot scan by running sequence 1 (see Basic Protocol 1).

\section{Sequence 4: Short $T_{\mathrm{R}}$ gradient echo timing bolus scan}

The timing of the contrast bolus can be critical. Image acquisition should occur during the first pass of the bolus, coinciding with arterial enhancement before arrival into adjacent veins. Ideally, the filling of central $k$-space should occur during peak arterial enhancement. Circulation times can vary widely among patients although bolus arrival times in the carotid arteries generally occur 15 to $20 \mathrm{sec}$ after initiation of the bolus. This can be calculated by using a timing run with a small test bolus of contrast agent measured by a single slice fast 2-D gradient echo sequence that acquires an image approximately every second over the duration of the circulating bolus. Some newer scanners are also capable of real-time "fluoroscopic MR imaging," which allows the contrast-enhanced MRA sequence to be manually triggered immediately after the fast 2-D sequence upon observation of peak arterial enhancement. For both injections, a power injector should be used to deliver the solutions at a rate of 2 to $3 \mathrm{ml} / \mathrm{sec}$.

5. Prepare the injector for a dose of 1 to $2 \mathrm{ml}$ of contrast agent to be followed by a 15 to $20 \mathrm{ml}$ bolus of saline flush. 
Table A1.3.5 Primary Clinical Imaging Parameters for Sequence 4 (Timing Bolus Scan)

Patient position

Scan type

Imaging plane (orientation)

Central slice or volume center

Echo time $\left(T_{\mathrm{E}}\right)$

Repeat time $\left(T_{\mathrm{R}}\right)$

Flip angle (FA)

Fields of view $\left(\mathrm{FOV}_{\mathrm{x}}, \mathrm{FOV}_{\mathrm{y}}\right)$

Resolution $(\Delta x, \Delta y)$

Number of data points collected $\left(N_{\mathrm{x}}, N_{\mathrm{y}}\right)$

Display matrix $\left(D_{\mathrm{x}}, D_{\mathrm{y}}\right)$

Slice thickness $(\Delta z)$

Number of slices

Slice gap

Number of acquisitions $\left(N_{\text {acq }}\right)$

Number of repetitions

Read direction

Slice locations

Scan time
Supine

2-D gradient echo

Transverse

Centered on the carotid bifurcation

$2.3 \mathrm{msec}$

$4.5 \mathrm{msec}$

$8^{\circ}$

$350 \mathrm{~mm}, 262 \mathrm{~mm}$

$1.37 \mathrm{~mm}, 1.36 \mathrm{~mm}$

256, 192

256,256

$10 \mathrm{~mm}$

1

N/A

1

60

Anterior-posterior

Carotid bifurcation or stenosis

$\sim 52 \mathrm{sec}$

Table A1.3.6 Primary Clinical Imaging Parameters for Sequence 5 (3-D CE TOF)

\begin{tabular}{ll}
\hline Patient position & Supine \\
Scan type & 3-D gradient echo \\
Imaging plane (orientation) & Coronal \\
Central slice or volume center & Centered on the carotid bifurcation \\
Echo time $\left(T_{\mathrm{E}}\right)$ & As short as possible (usually 2-3 msec) \\
Repeat time $\left(T_{\mathrm{R}}\right)$ & As short as possible (usually $4-8 \mathrm{msec})$ \\
Flip angle $(\mathrm{FA})$ & $10^{\circ}-45^{\circ}$ \\
Fields of view $\left(\mathrm{FOV}_{\mathrm{x}}, \mathrm{FOV}_{\mathrm{y}}\right)$ & $256 \mathrm{~mm}, 160 \mathrm{~mm}$ \\
Resolution $(\Delta x, \Delta y)$ & $1 \mathrm{~mm}, 1 \mathrm{~mm}$ \\
Number of data points collected $\left(N_{\mathrm{x}}, N_{\mathrm{y}}\right)$ & 256,160 \\
Display matrix $\left(D_{\mathrm{x}}, D_{\mathrm{y}}\right)$ & 256,256 \\
Slice thickness $(\Delta z)$ & $2-3 \mathrm{~mm}$ \\
Number of slices & $16-24$, depending on the coverage \\
Slab thickness & needed \\
Slice gap & $32-72 \mathrm{~mm}$, depending on the coverage \\
Number of acquisitions $\left(N_{\mathrm{acq}}\right)$ & needed \\
Read direction & 0 mm \\
Slice locations & 1 \\
Scan time & Cranial/caudal \\
\hline & Centered along long axis of vessel of \\
& interest to include vessel at all levels \\
& required \\
& $\sim 20-30$ sec \\
\hline
\end{tabular}

Extracranial Carotid Artery Disease 
6. Start sequence 4 according to the parameters in Table A1.3.5 prior to injection so that it runs continuously for $\sim 50 \mathrm{sec}$.

7. After $15 \mathrm{sec}$, start the injection so that the same data are collected prior to injection for baseline purposes.

8. Find the image in which there is a maximum signal. Some systems come with software to allow a region-of-interest plotted over all the sequence acquisitions. Obtain the time when the signal is at maximum. This time indicates the scan delay time.

A detailed estimation of the scan delay time is described in UNIT A10.1, Basic Protocol 2.

\section{Sequence 5: Short $T_{\mathrm{R}}$ 3-D gradient echo post-contrast scan}

9. Let the patient know that you are ready to start the main scan and instruct the patient to remain as motionless as possible.

10. Prepare the injector with a dose of 0.1 to $0.2 \mathrm{mmol} / \mathrm{kg}$ of contrast agent to be followed by a $15-$ to $20-\mathrm{ml}$ saline solution flush.

11. Inject the contrast agent and flush the saline solution. Wait for the scan delay time.

12. After obtaining the scan delay time, immediately start the scan according to the parameters given in Table A1.3.6 so that the center of $k$-space corresponds to the peak signal from the analysis in step 8.

The center of $k$-space should be used at the peak of the bolus. Preferably a centric ordered elliptic $k$-space coverage will be used.

Waiting too long to start the scan will result in high signal from veins as well as arteries.

13. If a more detailed scan is needed to better visualize the vessels and time permits, immediately collect a second, higher resolution scan by changing $N_{\mathrm{x}}$ from 256 to 512 and $N_{\mathrm{y}}$ from 160 to 320 .

See Haacke and Masaryk (1989) for a discussion of this high-resolution scan.

The patient can then be removed from the magnet and the data analyzed.

\section{IMAGING BLOOD VESSEL WALLS BY SPIN-ECHO MRA}

The inclusion of some sequences to study blood vessel walls after the vessels have been imaged initially by time-of-flight MRA (see Basic Protocol 1) and an abnormality (e.g., stenosis) has been discovered is likely to become commonplace in the future. The simplest approach for studying vessel-wall disease is to collect high-resolution images using a spin-echo (SE) or turbo-spin-echo sequence (Yuan et al., 1996; Raynaud et al., 1998). Although this is not yet a common procedure, it is anticipated that this will become part of a single overall imaging protocol for evaluating carotid artery disease. The entire Basic Protocol 2 takes $\sim 25 \mathrm{~min}$.

\section{Set up patient and equipment}

1. Repeat Basic Protocol 1, steps 1 to 10.

\section{Sequence 6: High-resolution spin-echo scan}

2. Run a spin-echo sequence using the parameters described in Table A1.3.7 to obtain vessel-wall information and general morphological information about the vessels and surrounding tissue. 
Table A1.3.7 Primary Clinical Imaging Parameters for Sequence 6 (2-D SE)

\begin{tabular}{|c|c|}
\hline Patient position & Supine \\
\hline Scan type & Spin echo \\
\hline Imaging plane (orientation) & Transverse \\
\hline Central slice or volume center & $\begin{array}{l}\text { Centered on the abnormality of } \\
\text { interest (commonly at the skull base or } \\
\text { carotid bifurcation) }\end{array}$ \\
\hline Echo time $\left(T_{\mathrm{E}}\right)$ & $15 \mathrm{msec}$ \\
\hline Repeat time $\left(T_{\mathrm{R}}\right)$ & $500-700 \mathrm{msec}$ \\
\hline Flip angle (FA) & $90^{\circ}$ \\
\hline Fields of view $\left(\mathrm{FOV}_{\mathrm{x}}, \mathrm{FOV}_{\mathrm{y}}\right)$ & $\begin{array}{l}256 \mathrm{~mm}, 200 \mathrm{~mm} \text { (or as small as } \\
\text { appropriate without significant } \\
\text { aliasing) }\end{array}$ \\
\hline Resolution $(\Delta x, \Delta y)$ & $0.5 \mathrm{~mm}, 1 \mathrm{~mm}$ \\
\hline Number of data points collected $\left(N_{\mathrm{x}}, N_{\mathrm{y}}\right)$ & 512,200 \\
\hline Display matrix $\left(D_{\mathrm{x}}, D_{\mathrm{y}}\right)$ & 512,512 \\
\hline Slice thickness $(\Delta z)$ & $3 \mathrm{~mm}$ \\
\hline Number of slices & $\begin{array}{l}10-20 \text {, if more slices are needed to } \\
\text { cover the region of interest, then the } \\
\text { scan can be repeated as necessary }\end{array}$ \\
\hline Slice gap & $0 \mathrm{~mm}$ \\
\hline Number of acquisitions $\left(N_{\mathrm{acq}}\right)$ & 1 \\
\hline Read direction & Anterior-posterior \\
\hline Slice locations & Centered about the abnormality \\
\hline Saturation pulses & $\begin{array}{l}\text { Yes; caudal and cephalic (cranial) } \\
\text { thick slabs to saturate both arteries and } \\
\text { veins using parallel saturation bands }\end{array}$ \\
\hline Fat suppression & Yes (for carotid dissection) \\
\hline Slice series & Interleaved \\
\hline Scan time & $\sim 2 \mathrm{~min}, 30 \mathrm{sec}$ \\
\hline
\end{tabular}

\section{COMMENTARY}

\section{Background Information}

Atherosclerosis of the carotid artery is an important medical condition because it is associated with strokes, the third-greatest cause of death in the United States. Obtaining detailed information about carotid artery stenosis (narrowing) can be critical to the determination of appropriate treatment, e.g., data from the North American Symptomatic Carotid Endarterectomy Trial (NASCET) indicated that in patients with symptomatic carotid stenosis of $>70 \%$, the incidence of stroke was lower in patients treated with surgery than in those receiving medical care only. The traditional means of imaging stenosis has been the invasive technique of carotid angiography. More recently, ultrasound has been suggested as a non-invasive alternative, but numerous studies have demonstrated that it is less accurate. Magnetic resonance angiography (MRA) is another non-invasive technique-based, like ultrasound, on the detection of flow through the artery. The related imaging technique of contrast-enhanced MRA, on the other hand, does not depend on the presence of significant blood flow and may, in the future, prove to be the most accurate means of assessing the vessel lumen. Both of these potentially complementary methods are presented in this unit (see Basic Protocol 1; see Alternate Protocol). Common indications for performing carotid MRA include transient ischemic attacks, stroke, neck injuries, suspicion of carotid dissection, neck tumors, tumor invasion of the carotid artery, carotid bruit on clinical exam, or abnormal ultrasound screening results prior to carotid bypass surgery. Other methods for imaging blood vessels include ultrasound and computed tomography angiography (CTA). Ultrasound provides good imaging results only for surface vessels, but can also
Extracranial Carotid Artery Disease 
give information about vessel-wall thickness. CTA does about as good a job as MRA but requires the use of ionizing radiation and a contrast agent.

\section{Critical Parameters and Troubleshooting}

Magnetic resonance angiography without contrast agents works on the principle of detecting enhanced signal from blood due to the inflow of fresh spins (see UNIT B7.3 for more technical details). For a given repeat time $\left(T_{\mathrm{R}}\right)$, the choice of flip angle determines not only the available signal from the blood but also the available contrast between the blood and surrounding tissue. The faster the flow, the larger the flip angle that can be used to optimize the signal from the blood and suppress the signal from background tissue. For small vessels, high resolution is key to creating a successful MR angiogram. This is also true for larger vessels if it is important to quantify the degree of stenosis. The actual application of the sequences presented in this unit may require some modifications depending on the age of the patient, the exact region being evaluated, and the time available for scanning. For example, newborns have very slow arterial flow, whereas in children aged 2 to 10 , flow is very fast, which makes it possible to increase the slab thickness without loss of vascular information. Elderly patients often have tortuous vessels and slow flow, possibly requiring contrast-enhanced MRA. Patients with tight stenoses and rapid flow (up to $4 \mathrm{~m} / \mathrm{sec}$ ) may demonstrate vessels with signal dropout, where contrast-enhanced imaging may also help. Artifacts in carotid artery imaging pose significant problems for the interpretation of results. Three common classes of artifacts are discussed below; for further information, any one of a number of standard texts, such as Potchen et al. (1992), may be consulted.

\section{Saturation artifacts}

As a patient ages, blood vessels can become tortuous and blood flow significantly slows relative to younger people. The winding of the vessels can lead to retrograde flow in those patients relative to the presumed upward direction and this can cause saturation on the 2-D TOF sequence (see UNIT B7.3). This is less of a problem with 3-D imaging, where a lower flip angle is used along with a spatially variable pulse to try and avoid this difficulty. Still, even 3-D images can suffer if the flow is too slow. Also, a shorter echo time $\left(T_{\mathrm{E}}\right)$ can often give better results because it has better flow compensation and reduced acceleration effects.

\section{Signal loss artifacts with gradient echo imaging}

Signal loss in the vessels themselves can be caused by insufficient velocity compensation. This can be improved upon by running a velocity compensated sequence with a shorter echo time. Significant signal loss throughout the image or in a large local region in gradient-echo imaging can be caused by the presence of small amounts of metals, especially those containing iron or nickel. Even iron particulates in clothing can lead to giant gaps in the image. Removing all clothing, jewelry, and mascara can help avoid this problem.

\section{Artifacts in post-contrast scanning}

Two major problems can occur. If the 3-D post-contrast scan is started too soon, only the edges of the arteries will enhance. If it is started too late, the veins may be as bright as the arteries making the viewing of the data in the MIP form very difficult. Ideally, when the timing is perfect, the arteries will be much brighter than the veins and only the edges of the veins will appear if at all.

\section{Anticipated Results}

The goal in studying the carotid vessels is to diagnose the degree of narrowing and, if possible, the damage to the vessel wall itself. Magnetic resonance imaging offers the necessary contrast to separate the vessels from other tissue. Conventional 3-D TOF scans are generally effective for visualizing the carotid arteries (see Fig. A1.3.2). With a resolution of $1 \mathrm{~mm}$ in all three directions and possibly higher for in-plane resolution, vessels are well delineated. With the better modern gradients allowing shorter echo times and less signal loss from dephasing, stenoses can be seen clearly. In principle, it is also possible to quantify the vessel lumen (i.e., the extent of stenosis), although approved software offering this feature is not yet widely available from equipment manufacturers. Using MRA, the carotid arteries can be imaged with a cross-sectional resolution of $\sim 0.5 \mathrm{~mm}$ by $0.5 \mathrm{~mm}$, with a slice thickness of $1 \mathrm{~mm}$. This is sufficient to obtain 10 to 20 pixels across the vessel lumen. This resolution then limits how accurately one can quantify the stenosis. The use of contrast agents to enhance resolution is now commonplace. These agents reduce the $T_{1}$ of the blood, leading to a larger signal from blood relative to all other
Intracranial Arterial Disease A1.3.11 
A

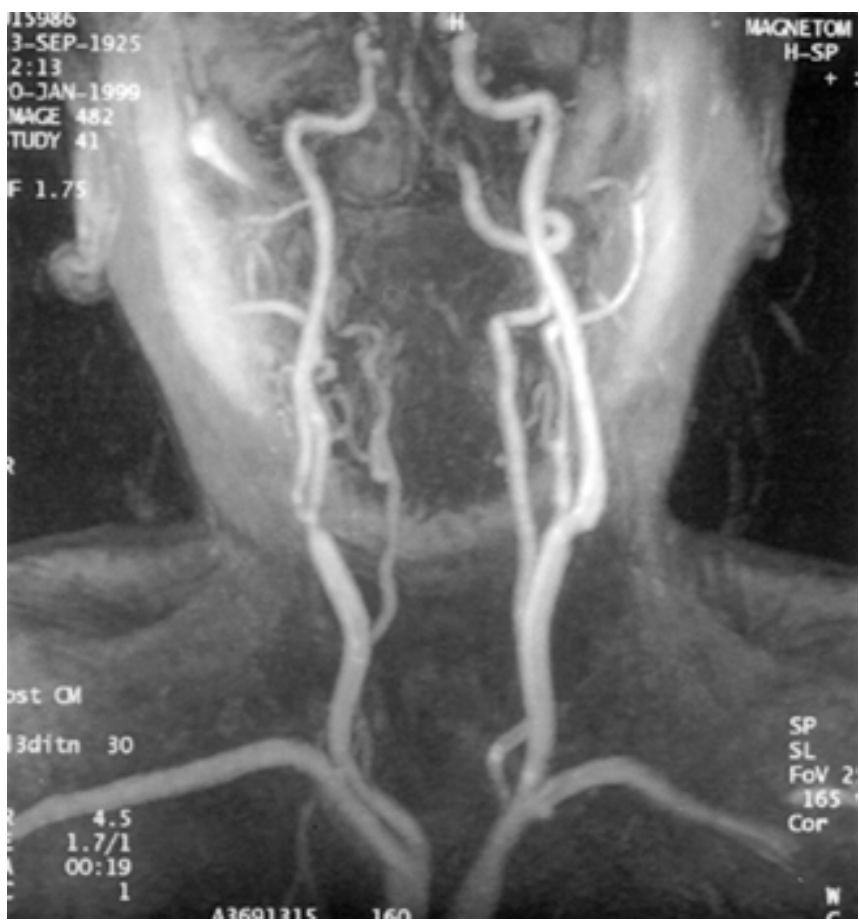

B

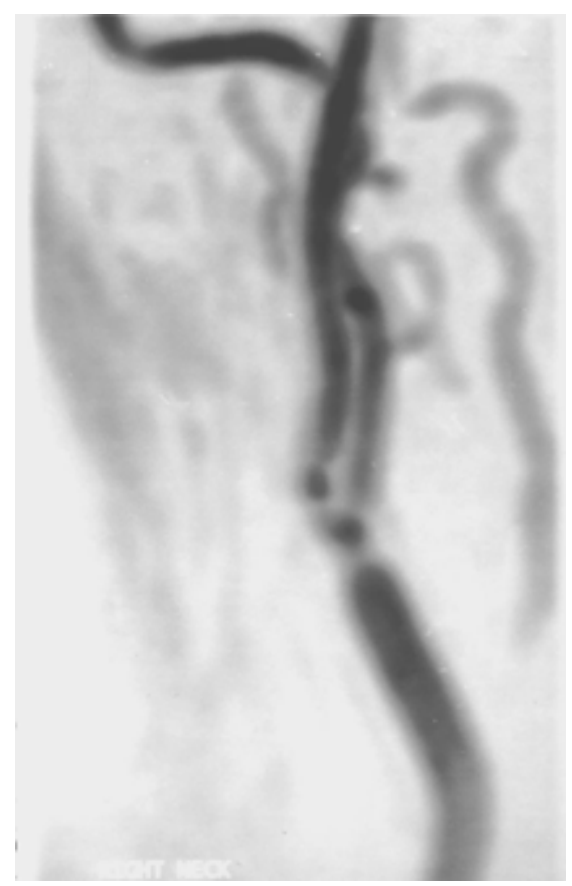

Figure A1.3.3 Images from a contrast-enhanced scan immediately after injection of the contrast agent. Data was acquired coronally so that all vessels could be seen in projection mode. The in-plane resolution is just high enough to show the diseased vessels. (A) A major stenosis is present on the right carotid and some abnormality on the left carotid. (B) A magnified view of the right carotid shows the latter problem more clearly.

Extracranial Carotid Artery Disease 
tissues independent of flow, so that the blood will be bright even in cases of slow or obstructed flow. Immediately post injection, only the arterial blood appears bright; after $\sim 30 \mathrm{sec}$, the signal from venous blood begins to enhance as well. The advantage of using a contrast agent is the increased coverage and the rapid imaging time (see Fig. A1.3.3). In-phase resolution is sufficient to visualize severe stenoses easily.

\section{Acknowledgments}

The authors would like to thank Glenn Foster, R.T., for reading this material and providing helpful comments. The authors would also like to thank Yingjian Yu for checking sequence parameters.

\section{Literature Cited}

Anderson, C.M., Edelman, R.R., and Tarshi, P.A. (eds.) 1993. Clinical Magnetic Resonance Angiography. Raven Press, New York.

Haacke, E.M. and Masaryk, T.J. 1989. The salient features of MR angiography. Radiology 173:611-612.

Haacke, E.M., Brown, R.W., Venkatesan, R., and Thompson, M.R. 1999. Magnetic Resonance Imaging: Physical Principles and Sequence Design. John Wiley \& Sons, New York.

Potchen, J.E., Haacke, E.M., Siebert, J.E., and Gottschalk, A. (eds.) 1992. Magnetic Resonance Angiography. C.V. Mosby, St. Louis.

Prince, M.R., Chenevert, T.L., Foo, T.K., Londy, F.J., Ward, J.S., and Maki, J.H. 1997. Contract enhanced abdominal MR angiography: Optimization of imaging delay time by automating the detection of contrast material arrival in the aorta. Radiology 203:109-114.

Raynaud, J.-S., Bridal, S.L., Toussaint, J.-F., Fornès, P., Lebon, V., Berger, G., and Leroy-Willig, A. 1998. Characterization of atherosclerotic plaque components by high resolution quantitative MR and US imaging. J. Magn. Reson. Imaging 8:622-629.

Shellock, F.G. 2001. Pocket Guide to MR Procedures and Metallic Objects. Lippincott-Raven, Philadelphia.
Wilman, A.H., Riederer, S.J., Huston, J., 3rd., Wald, J.T., and Debbins, J.P. 1998. Arterial phase carotid and vertebral artery imaging in 3-D contrast-enhanced MR angiography by combining fluoroscopic triggering with an elliptical centric acquisition order. Magn. Reson. Med. 40:24-35.

Yuan, C., Skinner, M.P., Kaneki, E., Mitsumori, L.M., Hayes, C.E., Raines, E.W., Nelson, J.A., and Ross, R. 1996. Magnetic resonance imaging to study lesions of atherosclerosis in the hyperlipidemic rabbit aorta. Magn. Reson. Imaging 14:93-102.

\section{Internet Resources}

http://www.mravvh.com

Vascular Visible Human Project, providing an overview of MRA imaging for different parts of the body.

http://www.mrisafety.com

Covers a number of important patient management issues related to MR imaging, including recommended safety procedures, a list of metallic implants that have been tested for MR compatibility, and a list of other sources on MR safety.

\section{Key References}

Potchen, E.J., Haacke, E.M, Siebert, J.E., and Gottschalk, A. 1993. Magnetic Resonance Angiography: Concepts and Applications. C.V. Mosby, St. Louis.

Contains detailed descriptions of the technical aspects of magnetic resonance angiography, as well as numerous clinical examples.

Shellock, F.G. 2001. Magnetic Resonance Procedures: Health effects and safety. CRC Press, Fla..

Covers a number of important patient management issues related to MR imaging, including recommended safety procedures, a list of metallic implants that have been tested for MR compatibility, and a list of other sources on MR safety.

Contributed by E. Mark Haacke

The MRI Institute for Biomedical Research St. Louis, Missouri

Daniel Kido and Karen Tong

Loma Linda University

Loma Linda, California
Intracranial Arterial Disease

A1.3.13 